\title{
A Time Series Sodar Simulator Based on Large-Eddy Simulation
}

\author{
Charlotte E. Wainwright, Phillip M. Stepanian, Phillip B. Chilson, \\ AND ROBERT D. PALMER \\ School of Meteorology, and Advanced Radar Research Center, University of Oklahoma, \\ Norman, Oklahoma \\ Evgeni Fedorovich AND JeREMY A. GiBBS \\ School of Meteorology, University of Oklahoma, Norman, Oklahoma
}

(Manuscript received 28 July 2013, in final form 17 November 2013)

\begin{abstract}
A sodar simulator capable of producing time series data emulating sodar signals has been developed and tested. The atmospheric fields used to populate the sodar simulator are taken from output of a large-eddy simulation code. The characteristics of the sodar (number and zenith angle of beams, beamwidth, transmit frequency, range resolution, etc.) are defined by the user to allow emulation of existing systems. The range of the reflected acoustic signal is calculated based upon a temperature-dependent speed of sound. Realistic acoustic background noise is simulated using filtered white noise. The raw acoustic time series data are processed using a Fourier transform to yield acoustic Doppler spectra, from which the radial velocities are calculated. The design of the simulator allows for the testing of and comparisons between various signalprocessing techniques and averaging periods. An example case of feeding the sodar simulator with large-eddy simulation data representative of a developing convective boundary layer is presented and discussed.
\end{abstract}

\section{Introduction}

Sodars have been widely used to study the atmospheric boundary layer (ABL) since their development in the 1970s (Kallistratova and Coulter 2004). The derived three-dimensional low-level wind fields that sodars can provide are used in air pollution monitoring and forecasting. The sodars are able to capture in detail boundary layer phenomena, such as the nocturnal lowlevel jet, convective structures in the daytime $\mathrm{ABL}$, and elevated inversions (Kallistratova and Coulter 2004). Sodars are also well suited to provide information about the structure of the ABL turbulence for the turbulence modeling community (e.g., Greenhut and Mastrantonio 1989). With continued growth of the interest in acoustic remote sensing, particularly for the wind energy industry, there is a need to develop improved scanning strategies and signal processing techniques to allow the maximum extraction of useful information from sodar returns.

Corresponding author address: Charlotte Wainwright, School of Meteorology, University of Oklahoma, 120 David L. Boren Blvd., Suite 5900, Norman, OK 73072.

E-mail: charlotte.wainwright@ou.edu
Despite their extensive employment in ABL studies, sodars do have some limitations. For instance, they typically cannot be used in highly populated areas due to the noise pollution they cause. The performance of sodars in complex terrain has been discussed (e.g., Neff 1988; Soler et al. 2003; Bradley 2008b) in connection with the level of surface heterogeneity that could affect sodar measurements. Since sodars largely make use of the Doppler beam swinging (DBS; Balsley and Gage 1982) technique to derive the three-dimensional wind field from three or more independent beams, there remain questions regarding the range of scales over which sodar observations of wind can be considered representative. This issue cannot be resolved through the use of a dense network of sodars in the field due to the acoustic interference that would result from close spacing of several sodars. Comparisons can be made between sodar-derived wind fields and those measured by an instrumented tower, although such comparisons are typically limited by the maximum height of an instrumented tower.

To investigate some of the outlined issues, we have developed a time series sodar simulator. It uses data from a numerical large-eddy simulation (LES) that 
generates a realistic turbulent flow field in the ABL. The simulator is designed such that the scanning parameters can be set to match different commercially available sodars. An additional benefit of the simulator is that it allows for repeated scanning of the same atmospheric fields using different scanning parameters and signal processing techniques, thus allowing for a meaningful comparison between methods.

Simulating the scanning of atmospheric fields by remote sensing instruments is a technique that has been used with much success in the radar meteorology community. Radar simulators, which can scan the output from high-resolution atmospheric models and largeeddy simulations, have been developed for weather radars (e.g., May et al. 2007; Cheong et al. 2008), millimeter-wavelength cloud radars (Clothiaux et al. 1996), boundary layer wind profiling radars (e.g., Muschinski et al. 1999; Scipión et al. 2009), threedimensional imaging radars (e.g., Yu and Palmer 2001; Cheong et al. 2004), and radars with dual-polarimetric scanning capabilities (e.g., Capsoni et al. 2001; Jung et al. 2008). Scattering of sodar signals has previously been modeled using a one-dimensional slab-based approach by Bradley (1999). To the authors' knowledge, this technique of simulating the scanning of full threedimensional atmospheric model output has never before been extended to acoustic remote sensing.

Section 2 describes in detail the algorithmic configuration of the sodar simulator. In section 3 , the moment simulator that is used for verification of the full time series sodar simulator is discussed. The LES code employed to generate the atmospheric fields sampled by the simulator is described in section 4 . Section 5 presents results from the initial testing of the simulator. Conclusions are drawn and future work is discussed in section 6 .

\section{Simulator setup}

\section{a. Overview of the simulator algorithms}

First we present a simple overview of the setup of the simulator, with further details on each step given in the following paragraphs. The sodar simulator ingests a succession of three-dimensional flow fields containing three resolved (in the LES sense) velocity components: $u, v$, and $w$; the resolved potential temperature of air $\Theta$; and the resolved water vapor mixing ratio $q$ from an LES. The simulator also ingests user-defined values for certain variables, detailed below. The location of each sodar beam is then calculated based upon the temperature-varying speed of sound (sections $2 b, c)$. Following this, the radial velocities and a proxy for the acoustic power are calculated at the beam locations.
Weighting functions are applied to the radial velocity and power (section 2d). The atmospheric absorption and acoustic backscattering cross section are next calculated at the beam locations (sections $2 \mathrm{e}, \mathrm{f}$ ). At this stage all the components are in place to calculate the phase and amplitude of the received complex acoustic signal.

For each beam, the complex acoustic signal is coherently summed across a number of beam points $b$ within the beam to provide a time series in range. The beams are divided into range gates of the appropriate length, and time series acoustic background noise is generated for each gate and added to the complex acoustic signal (section 2g). A fast Fourier transform (FFT) is performed on the complex noisy signal contained in that range gate. To process the synthesized signals, an average noise spectrum is generated for each beam and used to denoise the spectra at each range gate. The denoised Doppler spectra are finally processed to derive the radial velocity at each range gate and beam (section $2 \mathrm{~h}$ ). This process is repeated for the number of independent beams required and then the beam sequence is repeated until the desired runtime of the simulator has elapsed. Estimation of the three-dimensional wind field is performed on each beam sequence. Temporal averaging of the spectra to gain time-averaged wind field estimates is applied as postprocessing (section 2i).

An overview of the workflow of the time series simulator is presented in Fig. 1. Equations corresponding to each process are denoted in parentheses, and more detail on each of these processes is presented within the following subsections.

\section{b. Beam locations}

Many of the characteristics of the simulated sodar are defined by the user, allowing for the emulation of different sodar models and the testing of operating strategies. These include the placement of the sodar within the LES domain and the number of independent beam directions. The transmitted frequency, beamwidth, azimuth, and zenith angle of each beam are adjustable. The desired approximate vertical range resolution is also set by the user. The vertical range resolution should be chosen such that it is larger than the vertical resolution of the LES, for reasons detailed in section 5b. The pulse duration $\tau$ is calculated as

$$
\tau=\frac{2 \Delta r}{c_{\text {ref }}},
$$

where $\Delta r$ is the desired range resolution and $c_{\text {ref }}$ is a reference speed of sound of $340 \mathrm{~m} \mathrm{~s}^{-1}$ (corresponding to a temperature of $288 \mathrm{~K}$ for dry air). 


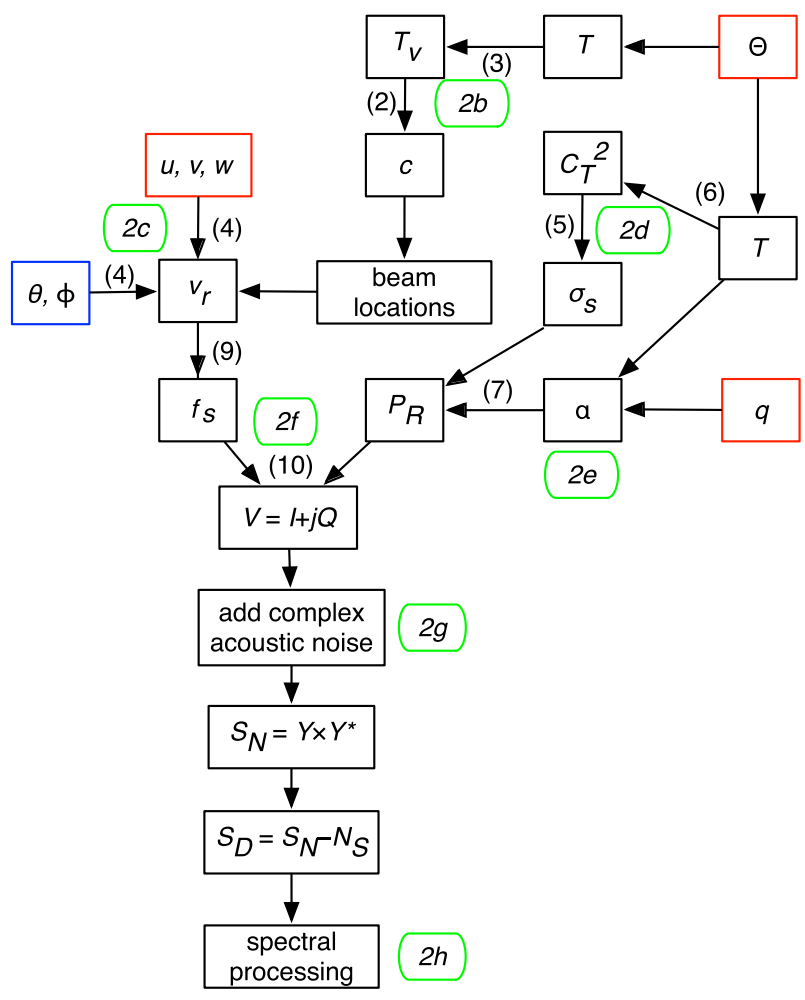

FIG. 1. An overview of the workflow of the time series simulator. The equations corresponding to the processes referred to are in parentheses. The section numbers covering each process are outlined in green. The red boxes indicate variables provided by the LES output, and the blue box indicates parameters defined by the user.

Acoustic waves do not travel at a constant speed. The speed of sound is far more sensitive to temperature variations than electromagnetic waves are; for the same temperature variations, the changes in refractive index are 1000 times greater for acoustic waves than for electromagnetic waves (Kallistratova 1997). The speed of sound $c$ varies with temperature as

$$
c=20.05 \sqrt{T_{v}},
$$

where $T_{v}$ is the virtual temperature in kelvins, and $c$ is in meters per second (May et al. 1990); $T_{v}$ is calculated from the ingested field of $\theta$ via

$$
T_{v}=\frac{T}{1-\frac{e}{p}(1-0.622)},
$$

where $p$ is the atmospheric pressure, $e$ is the vapor pressure, and $T$ is the air temperature in kelvins.

Owing to the temperature dependence of $c$, the speed of sound typically changes with height, causing a corresponding slight variation in the range resolution of a sodar with height, for a constant pulse length. This is worth noting, because a sodar operating without radio acoustic sounding system (RASS) capability typically measures the temperature at the instrument height only. The measured temperature is used to calculate the speed of sound, and the calculated speed $c$ is used to determine the time duration assigned to a particular height range. This will only assign correct range values under isothermal conditions. If the temperature change with height is essential, such as under conditions of strong radiative cooling/heating or within a strong inversion, then the reported measurement heights may be off by several meters (Fig. 2). For general atmospheric monitoring, this small difference between the reported and actual measurement height may not be significant, but it could be important for cloud base monitoring, for example.

To estimate the three-component wind vectors from the sodar, at least three independent and noncoplanar beams must be employed. This is typically achieved using a vertically oriented beam and two off-vertical beams that are horizontally mutually perpendicular. The DBS technique requires the assumption of horizontal homogeneity in the wind fields. This assumption is typically invalid, and as a result, the wind components derived from the sodar do not represent the winds directly above the sodar but include contributions from the sampling domain bounded by the off-vertical beams. Comparing simulator-derived winds to those taken directly from the LES requires consideration of the effects of spatial sampling. As the areal extent of the sampling domain increases with height, straightforwardly comparing sodar-derived winds to LES winds over a vertical column above the sodar point location will show increasing errors with height. To combat the effect of spatial sampling, a moment simulator has been developed. The moment simulator uses the LES wind fields to calculate the radial velocity projected along each beam at the prescribed range resolution matching that of the time series simulator.

The number of calculation points contained within each beam is defined as $b \times b \times n \times M$, where $b$ is the number of calculation points in each horizontal direction, $n$ is the number of layers of calculation points contained within one range gate, and $M$ is the number of range gates within each beam. This feature is illustrated for one beam in Fig. 3. While each horizontal layer of beam points $n$ is depicted as planar in Fig. 3 for simplicity, each point in the layer is equidistant from the sodar location; hence, the layer is of convex shape. Appropriate values for $b$ and $n$ should be chosen with the LES resolution in mind, as this determines the maximum number of points available for interpolation. 

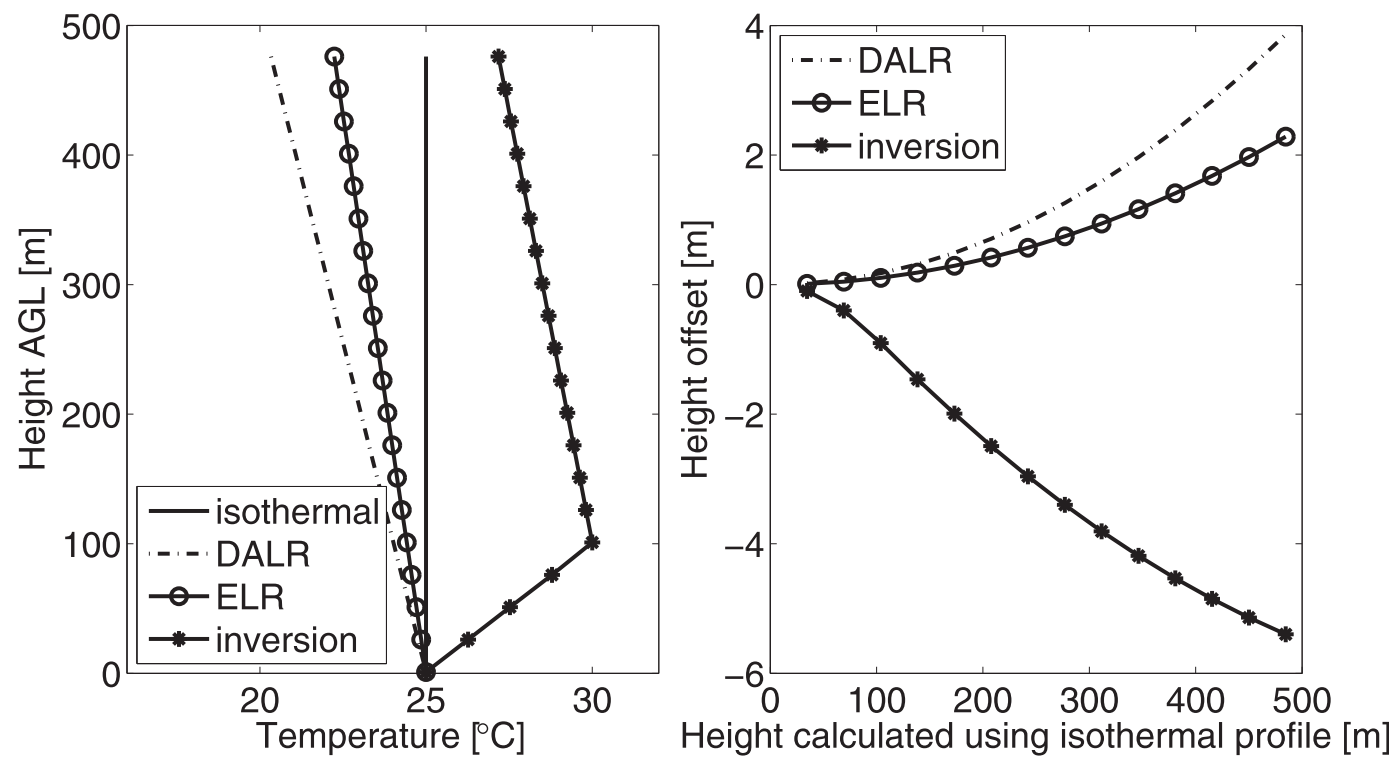

FIG. 2. (left) Four example vertical temperature profiles: isothermal, dry adiabatic, standard atmosphere, and a low-level inversion. (right) Offset in actual height of range gates for the different temperature profiles if the isothermal profile is assumed.

\section{c. Radial velocity}

Once the spatial location of each beam point has been determined, the radial velocities at those points are determined. The velocity components are first calculated at the beam points by using trilinear interpolation on the original fields of $u, v$, and $w$ from the LES resolved on an $X \times Y \times Z$ numerical grid. The velocity components are also interpolated in time between concurrent LES snapshots, to allow for spatial coherence between range gates. The temporal interpolation is done using the time durations assigned to each beam point during range determination.

The radial velocity is determined from the spatially interpolated velocity components as

$$
v_{r}(\theta, \phi)=u \sin \theta \sin \phi+v \sin \theta \cos \phi+w \cos \theta,
$$

where $\phi$ and $\theta$ are the azimuth and zenith angles of each beam point, respectively.

\section{d. Weighting functions}

Two types of weighting functions are used in the simulator. The function of the first type weights the radial velocity values based upon their location within the beam and within the range gate. The beam-weighting function employed is a multivariate normal distribution with a mean of the center of the beam in the $x$ and $y$ directions, and a standard deviation of the beamwidth. Points near boresight have the maximum value of $0 \mathrm{~dB}$, with the value decreasing to $-3 \mathrm{~dB}$ at specified beamwidth distance. The range-weighting function is based on an extended cosine pulse shape and also uses a maximum value of $0 \mathrm{~dB}$ at the center range of each beam and $-3 \mathrm{~dB}$ at the extremities of each range gate. The beamand range-weighting functions are combined to yield one single volumetric weighting function with values ranging from 0 to $-6 \mathrm{~dB}$. Weighting function of the second type is based upon the backscattering intensity at each beam point. The acoustic backscattering cross section is calculated as

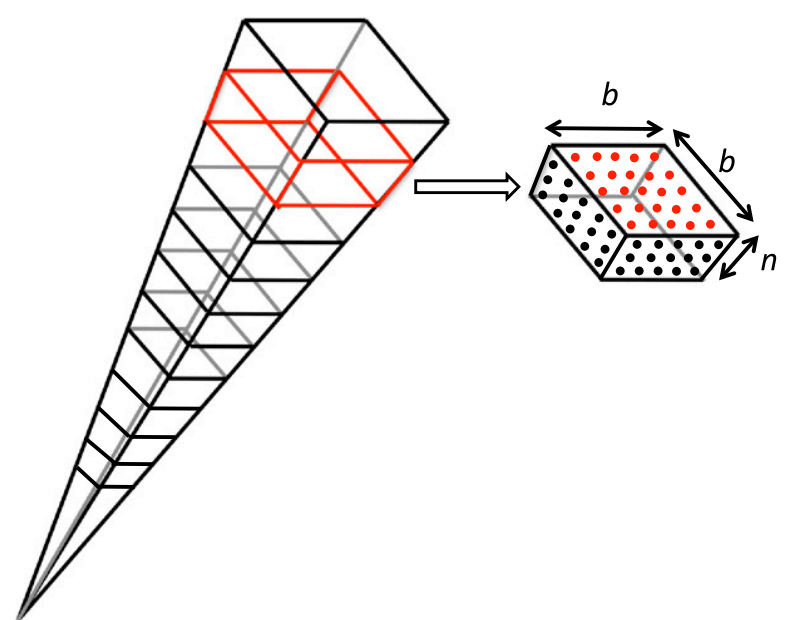

FIG. 3. The calculation points contained within each beam. (left) The single range gate outlined in red is shown (right) in detail. Each range gate contains $b \times b \times n$ points at which calculations are performed. Each of $n$ layers of beam points contains points that are equal in range from the sodar location. Thus, each layer is convex in shape, while depicted as planar here for simplicity. 


$$
\sigma_{s}=4 \times 10^{-3} \lambda^{-1 / 3} \frac{C_{T}^{2}}{T^{2}}
$$

(following Tatarskii 1971), where $\lambda$ represents the transmit wavelength of the sodar, which for the presented simulation is $0.15 \mathrm{~m}$. The structure function parameter for temperature is calculated using

$$
C_{T}^{2}=\frac{\left\langle[T(\mathbf{r}+\boldsymbol{\delta})-\mathrm{T}(\mathbf{r})]^{2}\right\rangle}{|\boldsymbol{\delta}|^{2 / 3}},
$$

where $\mathbf{r}$ is a position vector and $\boldsymbol{\delta}$ is the separation vector, and $\langle\cdot\rangle$ indicates averaging across all the difference pairs. The use of (5) is justified at scattering lengths within the inertial subrange, where the turbulence is assumed to be isotropic and homogeneous (Tatarskii 1971). The difference term is evaluated for all possible pairs of $T$ values within a cube of equal size to the LES resolution and then normalized by the distance $\boldsymbol{\delta}$, which here is equal to the linear size of the LES grid cell (see section 4). These pairs are then averaged to give one $C_{T}^{2}$ value for each grid box, which is interpolated to the beam locations in both space and time. The second weighting function uses $C_{T}^{2} / T^{2}$ as a proxy for power. Weighting the radial velocity values with this power proxy ensures that the average radial velocity value for the range gate is weighted more heavily toward the most acoustically reflective points.

\section{e. Atmospheric absorption}

The intensity of the acoustic signal decreases as it travels through the atmosphere due to a number of factors, including losses caused by spherical spreading, scattering of the acoustic signal outside of the beam volume, and atmospheric absorption. The power loss due to spherical spreading is proportional to $1 / r^{2}$ and will be included in the calculation of the complex acoustic signal directly, as shown below in (7) and (10). Losses due to scattering out of the beam are neglected, as they were shown by Pan (2003) to be small in comparison to losses due to atmospheric attenuation for typical sodar operating frequencies. The loss due to atmospheric absorption $\alpha$ depends on the temperature, pressure, humidity, and transmitted frequency. We calculate $\alpha$ according to ISO (1993). The formula for $\alpha$ is not repeated here for brevity, but a typical value for the implemented case is $0.003 \mathrm{~dB} \mathrm{~m}^{-1}$.

\section{f. Complex acoustic signal}

The power of the acoustic signal after time $\tau$ at each beam point is modeled as

$$
P_{R}=P_{t} G A_{e} \sigma_{s} \frac{c \tau}{2} \frac{e^{-2 \alpha r}}{r^{2}},
$$

where $P_{T}$ is the transmitted power, $G$ is the antenna transmitting efficiency, $A_{e}$ is the effective area of the antenna, $\sigma_{s}$ is the backscattering cross section (5), $\alpha$ is the atmospheric absorption, and $r$ is the range (Little 1969). The phase of the signal at each beam point is calculated as

$$
\Phi=\exp \left[-j 2 \pi\left(f_{T}+f_{s}\right) t\right]
$$

where $f_{T}$ is the transmitted frequency and $f_{s}$ is the frequency shift due to nonzero radial velocity, calculated as

$$
f_{s}=\frac{2 f_{T} v_{r}}{c}
$$

Once the phase and amplitude of the signal are combined at each beam point to give

$$
V=I+j Q=\sqrt{P_{R}} \exp \left[-j 2 \pi\left(f_{T}+f_{s}\right) t\right],
$$

the signal that is coherently summed across the $b \times b$ beam points at each individual range. This provides a complex time series covering the entire range of each beam. The time series is then divided into height batches for each range gate for further signal processing.

\section{g. Acoustic background noise}

Acoustic background noise measured by sodars is not easily characterized and contains components due to localized effects, such as traffic, and echoes from nearby stationary objects. Bradley (2012) examined measured noise signals, separating sodar self-noise from external noise, and found that peaks in the measured noise spectrum were of unknown origin, while the external noise between these peaks followed a $1 / f$ frequency dependence. In this study the background noise is modeled as pink, such that it follows the $1 / f$ frequency dependence seen by Bradley (2012).

To model the pink noise, white noise is first generated and a filter is then applied to create the $1 / f$ roll-off that characterizes pink noise. Taking the FFT of the pink time series noise provides the noise spectrum. An example noise spectrum is shown in the left panel of Fig. 4. To emulate a physical sodar, a noise spectrum (denoted $N_{S}$ ) is generated prior to each new set of measurements. The same filter is used to generate instantaneous pink time series noise signals that are added to the complex time series signal within each range gate. Taking the FFT of the time series signal with added noise produces 

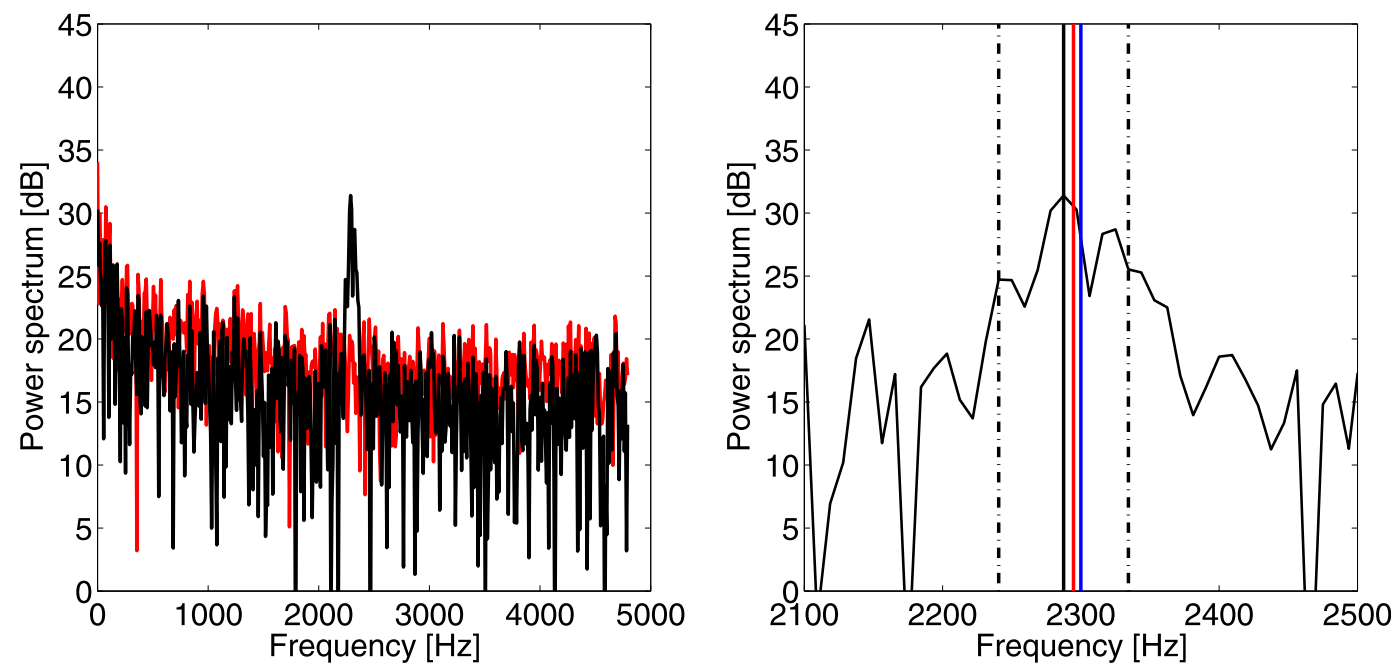

FIG. 4. (left) Example complete frequency spectrum with included noise output by the simulator for an off-vertical beam at a range of $165 \mathrm{~m}$ (black line). The generated noise spectrum is shown in red. (right) Illustration of the spectral processing. Points within a range of $2 m+1$ frequency bins are used to calculate the Doppler frequency. Here, $m=5$ and points in between the black dashed lines are included. The Doppler frequency used to calculate the radial velocity is marked in red. The transmitted frequency is marked by the blue line.

the noisy Doppler spectrum (shown in black in Fig. 4a). This completes the simulation of the signal.

\section{h. Spectral processing}

To process the synthesized signals, at each range gate and beam, an FFT is taken of the complex time series signal within that range, including both the backscattered acoustic signal and the background noise described in the previous section. Multiplying the result of the FFT $(Y)$ by its conjugate $\left(Y^{*}\right)$ gives the Doppler power spectrum, $S=Y \times Y^{*}$, for each range gate. The signal processing is performed on a gate-by-gate basis unlike a physical sodar, which typically operates on a full vertical time series profile at once. The single-gate processing was chosen purely to minimize computational expense in the model. Once a full scan containing all the range gates and all beams is completed, a single separate noise spectrum $N_{S}$ is generated. This single noise spectrum emulates the noise spectra that a sodar measures prior to each scan.

The single noise spectrum is subtracted from the noisy Doppler spectrum $S$ at each range gate to give an effective denoised Doppler spectrum $S_{D}$ (illustrated by the black line in Fig. 4a). The same $N_{S}$ is used for each range gate and beam within one scan. This allows us to best emulate a physical sodar, which uses the measured noise spectra obtained before each scan to remove noise from all the received Doppler spectra within the scan.

Further signal processing is performed on $S_{D}$. One of the benefits of the simulator is that it allows for the testing of different signal processing techniques on the same atmospheric fields, enabling a fair comparison of the different techniques.

Several signal processing techniques have been tested. The one used in this study is outlined in Bradley (2008a) and is illustrated in Fig. 4b. First, the peak of the denoised Doppler spectrum is identified (vertical black line of Fig. 4b). The frequency range to be used for determination of the frequency shift is defined by a number $2 m+1$ frequency bins, centered upon the identified peak, where $m$ is a positive integer. In this study we used $m=5$. The bounds of this frequency range are marked in Fig. 4b by dashed black lines. The frequency shift within these bounds is determined by calculating the first moment of the spectrum via the use of a maximum likelihood estimator that models the frequency spectrum as normally distributed. Only spectra within the range bounded by frequencies $2 m+1$ are used in the maximum likelihood estimator. This is done to reduce the influence of background noise on determination of the frequency shift. The frequency identified as the first moment is shown on Fig. 4b in red. The frequency shift for that range gate is simply the difference between the first moment and the transmitted frequency.

The radial velocity for each range gate is retrieved by rearranging (9) as

$$
v_{r}=\frac{f_{s} c}{2 f_{T}},
$$

where $c$ is calculated from (2) using the mean value of temperature for beam points located within the considered range gate. 


\section{i. Postprocessing}

If three or more independent noncoplanar beam directions are used, then the wind components $u, v$, and $w$ can be retrieved from the radial velocity values. Typically, either three or five independent beams are used. The radial velocities are related to the wind components through (4), which for a five-beam retrieval can be written as the system $\mathbf{C u}=\mathbf{v}_{\mathbf{r}}$, where

$$
\begin{aligned}
& \mathbf{C}=\left[\begin{array}{ccc}
\sin \theta_{1} \sin \phi_{1} & \sin \theta_{1} \cos \phi_{1} & \cos \theta_{1} \\
\sin \theta_{2} \sin \phi_{2} & \sin \theta_{2} \cos \phi_{2} & \cos \theta_{2} \\
\sin \theta_{3} \sin \phi_{3} & \sin \theta_{3} \cos \phi_{3} & \cos \theta_{3} \\
\sin \theta_{4} \sin \phi_{4} & \sin \theta_{4} \cos \phi_{4} & \cos \theta_{4} \\
\sin \theta_{5} \sin \phi_{5} & \sin \theta_{5} \cos \phi_{5} & \cos \theta_{5}
\end{array}\right] \\
& \mathbf{u}=\left[\begin{array}{c}
u \\
v \\
w
\end{array}\right] \text { and } \quad \mathbf{v}_{\mathbf{r}}=\left[\begin{array}{c}
v_{r 1} \\
v_{r 2} \\
v_{r 3} \\
v_{r 4} \\
v_{r 5}
\end{array}\right]
\end{aligned}
$$

where $\phi_{i}$ and $\theta_{i}$ are the azimuth and the zenith angle for beam $i$, respectively (Palmer et al. 1993). Once the calculated radial velocities are known, the wind components can be derived using the least squares solution of the matrix equation

$$
\mathbf{u}=\left(\mathbf{C}^{\mathbf{T}} \mathbf{C}\right)^{-1} \mathbf{C}^{\mathbf{T}} \mathbf{v}_{\mathbf{r}} .
$$

One of the advantages of the simulator is that it allows for a variety of averaging periods to be used on the same data, thus enabling an examination of the effect of the averaging period on the derived wind fields. The time averaging is performed by adding each individual Doppler spectrum within the desired averaging period, and recalculating the Doppler velocity based on the technique depicted in Fig. 4.

\section{Moment simulator}

To evaluate the performance of the sodar simulator, we wish to compare the derived three-component wind field back to the original wind field from the LES output. We chose to compare the results in two separate ways. The first way is to compare the estimated wind field from the simulator with wind values taken directly from the LES output. This would be equivalent to comparing winds from a sodar in a field campaign with winds measured on an instrumented tower (with the neglect of instrument error). One issue that can arise with this validation method is that it is difficult to separate the differences due to the spatial continuity assumption inherent in the DBS technique from the differences due to the spectral processing. To help separate these differences, we also estimate the wind fields using a so-called moment simulator, briefly described in this section.

The moment simulator uses the same beam locations and weighting as the time series sodar simulator, but it does not perform the spectral processing; rather, the radial velocities contained within one range gate are individually power weighted using $C_{T}^{2} / T^{2}$ and then simply averaged to gain a representative value for that gate. Thus, a comparison of the wind fields estimated by the moment simulator and the virtual instrumented tower would expose the differences that result solely from the spatial averaging the DBS technique implies. This is a useful technique because, as stated by Antoniou et al. (2003), there is no feasible way to make point and volume measurements directly comparable without distributing many point measurement devices throughout the volume encompassed by the sodar. While the moment simulator does not allow for a direct comparison of results between the point and volume measurements, it does allow for investigation of the biases between these measurement approaches, which is useful in itself. The challenges of comparing sodar- and instrumented mast-derived winds are investigated thoroughly by Bradley (2013), who states that the inherent difference between vector and scalar averaging causes an average root-mean-square (RMS) error in the measured winds of around $2 \%$. Use of the DBS technique is based on the assumption that each of the wind components $u, v$, and $w$ are constant across the region encompassed by the beam locations. Invalidity of this assumption caused by nonuniform vertical velocities or horizontal shear across the observed region can result in a bias in the wind estimates (shown for wind profilers by Koscielny et al. 1984).

Many field experiments have been performed to verify wind field measurements by sodars. Crescenti (1997) summarized many of the earlier studies that compared sodar-estimated winds with those from cup/vane anemometers, sonic anemometers, and lidars. It was concluded that Doppler sodars can reproduce reliable profiles of the mean wind speed and direction, with homogeneity in the wind fields leading to better comparisons between instruments. Inhomogeneity in the boundary layer adds complication and hampers interpretation of the resulting wind fields (Crescenti 1997). These effects can be examined by intercomparison of the winds from the virtual instrumented tower (LES) and the moment simulator. 


\section{Large-eddy simulation data}

The sodar simulator ingests turbulent flow fields generated by an LES code. For this study we populated the simulator with data from the University of Oklahoma LES (OU-LES, Fedorovich et al. 2004a). The simulation reproduced a developing convective boundary layer and was performed in a domain of size $1.28 \mathrm{~km} \times 1.28 \mathrm{~km} \times 1.5 \mathrm{~km}$ with a uniform grid spacing of $\Delta x=\Delta y=\Delta z=5 \mathrm{~m}$. The lowest model level was located at $2.5 \mathrm{~m}$ above ground level. The employed subgrid turbulence closure scheme is based upon Deardorff (1980). The LES output used for this study corresponds to 14001500 UTC 31 May 2009, in a domain centered upon the Atmospheric Radiation Measurement Program Southern Great Plains (ARM SGP) site in Lamont, Oklahoma. The OU-LES was nudged incrementally at each time step (about $1 \mathrm{~s}$ ) using vertical profiles of virtual potential temperature, water vapor mixing ratio, and the horizontal wind components from the Rapid Update Cycle (RUC; Benjamin et al. 1994), as described in Gibbs et al. (2011). The surface fluxes were prescribed using temporally interpolated sensible and latent heat fluxes from the eddy correlation flux measurement system (ECOR; Cook and Pekour 2008) at the ARM SGP site. The LES output fields that are used in the simulator are the threedimensional fields of potential temperature $\Theta$; the wind components, $u, v$, and $w$; and the water vapor mixing ratio $q$.

The OU-LES has been tested extensively for the convective boundary layer (CBL) conditions. Comparisons with experimental and numerical CBL data have shown that it can realistically reproduce mean flow and turbulence structure for clear CBL conditions (Fedorovich et al. 2001, 2004b; Botnick and Fedorovich 2008). The OU-LES output has previously been tested within the framework of a boundary layer radar simulator (Scipión et al. 2008), which allowed for testing of different scanning strategies and signal processing techniques for boundary layer radars, much like the present study does for acoustic remote sensors.

\section{Examples of using the sodar simulator}

\section{a. Experiment description}

The sodar simulator has been tested on output from the LES of the developing convective boundary layer (see section 4). The simulator has many user inputs that can be tailored to mirror existing systems. For this experiment, the parameters were tailored to match a Metek PCS.2000 sodar operated by the University of Oklahoma.

For the test, a subset of LES output data from the simulation described in section 4 of size $400 \mathrm{~m} \times 400 \mathrm{~m} \times 600 \mathrm{~m}$ was used. One hour of data was used with the simulated fields reported every $3 \mathrm{~s}$. The transmitted sodar acoustic frequency was set to $2300 \mathrm{~Hz}$. The maximum range was set to $500 \mathrm{~m}$ above ground level (AGL). The minimum range is not directly defined by the user but is rather determined from a defined transmit-to-receive delay that is set at $0.1 \mathrm{~s}$. This resulted in an effective minimum range of $26 \mathrm{~m}$. The range resolution was $11.6 \mathrm{~m}$ and 40 range gates are simulated. Five beams were used with the four off-vertical beams at $72.5^{\circ}$ elevation angle, and the beamwidth was $10^{\circ}$. The azimuth angles of the four offvertical beams were multiples of $90^{\circ}$. The fields of $u, v, w$, and $\Theta$ were updated with each new beam direction at 3-s intervals to give a complete set of measurements every $15 \mathrm{~s}$. This timing is typical for the operational sodar we are simulating in this study. The number of points that are equidistant in range within each beam $(b \times b)$ is set to $5 \times$ 5 . The number of ranges $n$ contained within each range gate is set to 640 , which equates to a sampling frequency close to $39000 \mathrm{~Hz}$.

A sample of the numerically simulated CBL flow is illustrated in Figs. 5 and 6. The boundary layer depth at the early time of the simulation is around $300 \mathrm{~m}$, as indicated in Fig. 5. The horizontal wind components $u$ and $v$ are both seen to increase in magnitude above the boundary layer. Beneath $300 \mathrm{~m}$, we see a pattern of updrafts (positive $w$ ) and downdrafts (negative $w$ ), with generally small magnitudes of $w$ above the CBL top. Figure 6 illustrates the strong spatial variability of $u, v$, and $w$ within the boundary layer, and it is clear that the assumption that the wind components are homogeneous across the area encompassed by the five sodar beams is invalid.

\section{b. Results}

Results based on the radial velocity calculations for the five beams of the sodar simulator are shown in Fig. 7. The top panel shows a time-averaged vertical profile of the RMS difference in the radial velocity of each beam calculated by spectral processing as compared to that from the moment simulator. One would expect the RMS difference to increase with height due to the effect of the assumption of spatial homogeneity inherent in the DBS technique over an increasingly large area. However, this does not seem to be the case. We see that heights with increased RMS differences in radial velocity - namely, close to the surface and at heights close to the boundary layer top-are regions that exhibit increased spatial heterogeneity of the three-dimensional wind components. One may expect that for all heights the RMS difference between the moment and spectrally processed radial velocities would tend to zero over a sufficiently long averaging period. We indeed see the 


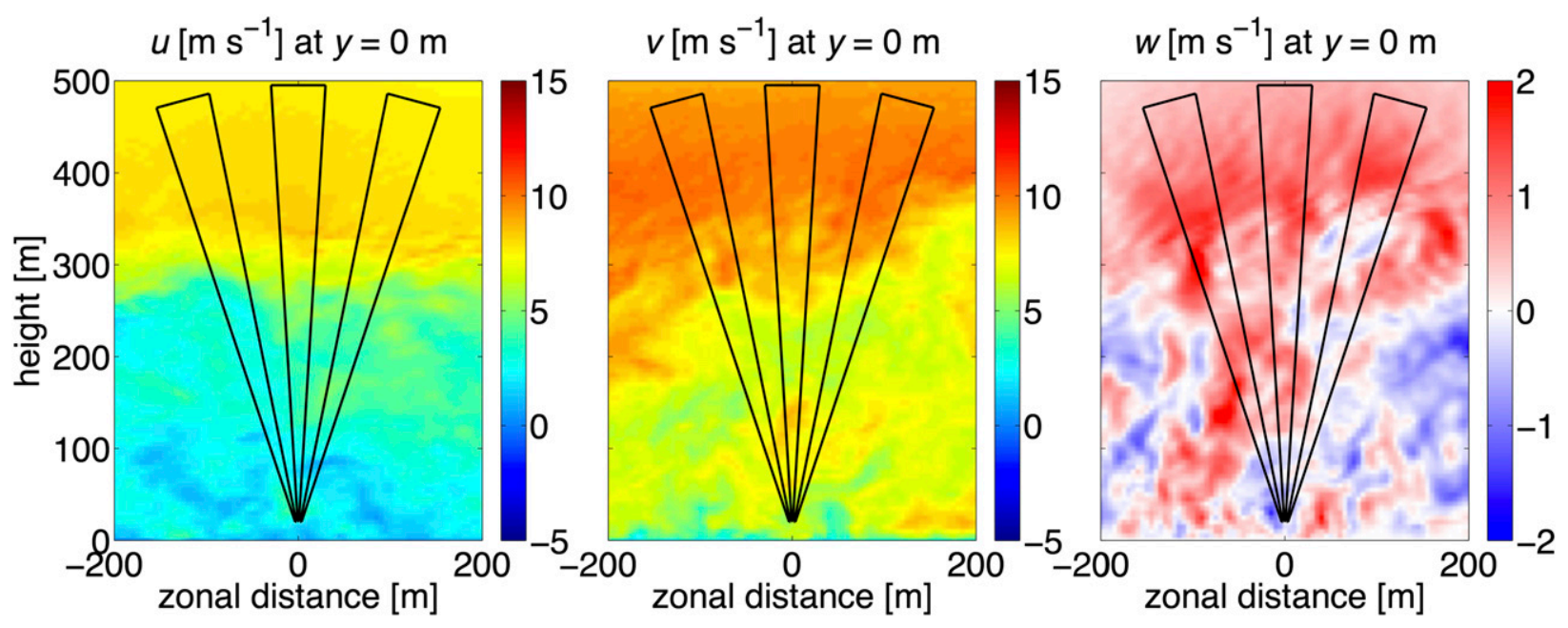

FIG. 5. Snapshots of the LES wind component fields at $y=0 \mathrm{~m}$. The areas encompassed by beams 1, 3, and 4 are overlaid. Presented data illustrate the failure of the assumption that the three wind components are constant across the region defined by the beam locations.

RMS radial velocity difference decreasing with the averaging period, but there seems to be a lower bound on the RMS difference for some beams and heights. This is most noticeable for beam 2 (the second panel in Fig. 7). Particularly for heights between 300 and $350 \mathrm{~m}$, the RMS difference does not significantly decrease as the averaging period increases over $5 \mathrm{~min}$, indicating a systematic bias of some kind.

The cause of this bias was thoroughly investigated. It was found that the highest RMS differences in radial velocity were collocated with range gates over which the radial velocity changed the most over the height within that range gate. If the radial velocity changes linearly (or almost linearly) with height within the range gate, then this introduces a linear variation in the frequency shift $f_{s}$ in (8), resulting in the complex voltage

$$
V=\sqrt{P_{R}} \exp \left\{-j 2 \pi\left[f_{T}+\left(f_{s}+A t\right)\right] t\right\},
$$

where $A$ represents the frequency slope caused by the linear change in radial velocity over the depth of the range gate. Taking the FFT of the signal in (13) does not result in a spectral peak located exactly at $f_{T}+f_{s}+A / 2$, which would be the instantaneous frequency at the central time moment within the FFT window, but results in smearing of the peak in the FFT and phase distortion (Masri and Bateman 1995).

A fundamental assumption when interpreting the FFT results is that the signal statistics (up to second order) are wide-sense stationary, that is, the signal is stationary over the period that the FFT is taken. For sinusoidal signals of the type considered in (10), it is assumed that the amplitude and frequency remain constant within the FFT window (in our case, the FFT window corresponds to the time interval for which the signal is within one range gate). The introduction of linear (or nonlinear) frequency modulation obviously invalidates this assumption. However, the FFT is the method used in most operational sodars.

This frequency shift is not typically problematic for operational sodars, as the perfectly linear frequency modulation in the simulator is a result of the trilinear interpolation of $u, v$, and $w$ to the beam points. For an operational sodar, the background and instrument noise would typically overshadow the linear radial velocity variation. For the sodar simulator, the effect of linear frequency modulation is more marked at lower heights, where the sodar beamwidth encompasses relatively few LES grid points. At higher levels there is rarely perfect linear frequency modulation due to radial velocity contributions from an increased number of LES grid points, which mitigates the frequency modulation effect to some degree. For this reason, it is recommended that LES output of sufficiently high spatial resolution to provide contributions from more than two grid points, even at the lowest range gates, is used.

One possible solution to overcome the linear variations in radial velocity is to include a contribution from the subgrid turbulence kinetic energy $E$ in the threedimensional wind components, as has been done for radar simulators, (e.g., Cheong et al. 2008; Scipión et al. 2009). The authors tested this idea within the time series simulator, but it was found to introduce large errors into the simulation results. Investigation into the cause of the subsequent errors revealed that at heights close to the surface and the boundary layer height, values of $E$ were 

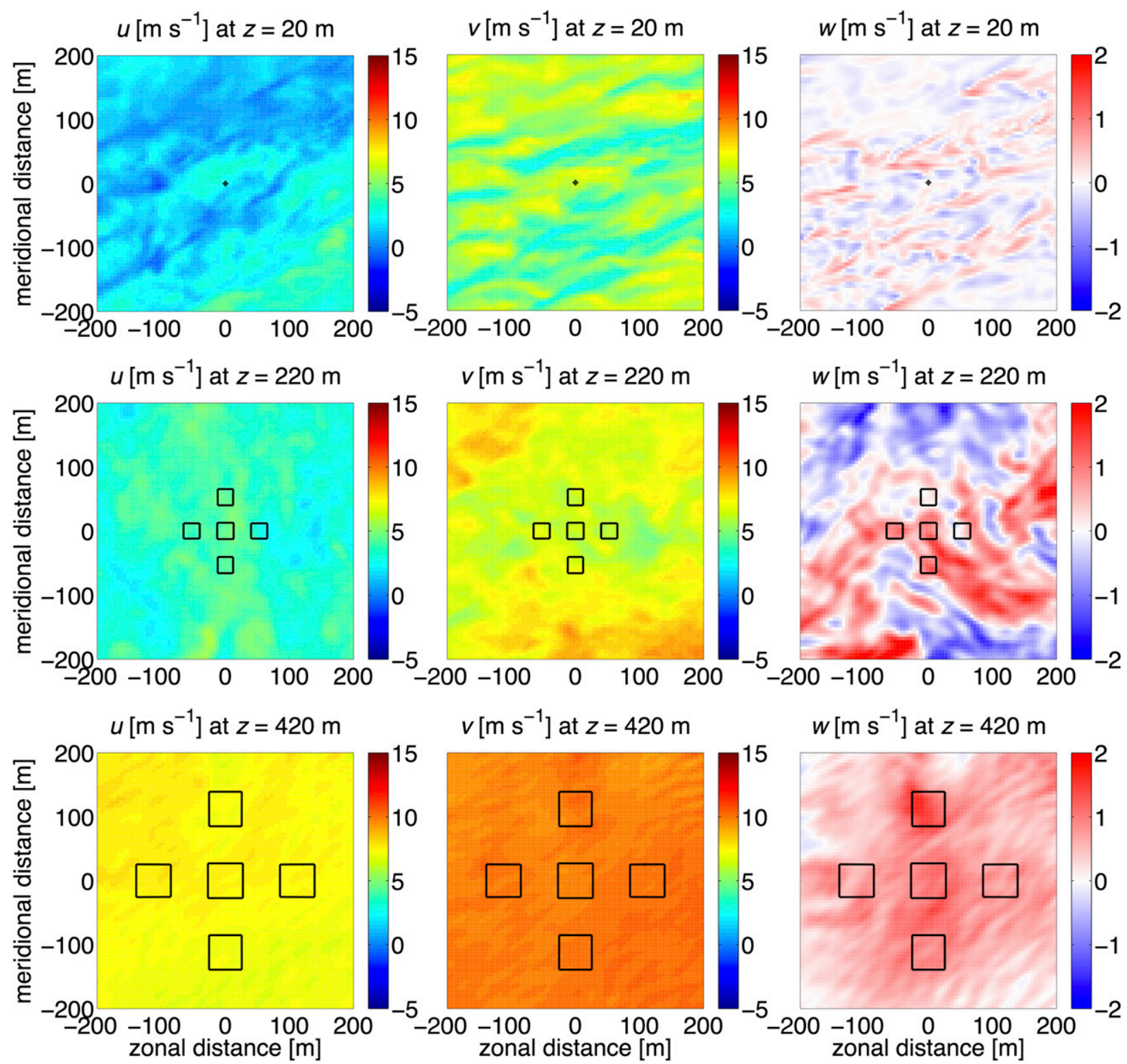

FIG. 6. Snapshots of the LES wind component fields at 20,220, and $420 \mathrm{~m}$ above ground level. Wind fields shown are from $30 \mathrm{~min}$ into the 60-min simulation used for this experiment. The areas encompassed by each beam at the respective heights are overlaid.

as large as the resolved wind components, resulting in large errors in the spectrally derived radial velocity.

In interpolating $E$ down to a finer scale, we ignore the inherent scale dependence of the resolved velocity field and $E$ in LES. One would expect that on a spatial scale comparable to the beam point spacing used in the simulator $(0.018 \mathrm{~m}$ in the vertical), the subgrid contribution would be significantly lower than the resolved contribution. We suggest that future work include an investigation into the scaleability of resolved velocity components and $E$ down to small scale. This would allow the inclusion of contributions from $E$ in the radial velocity, which would compensate for the linear variation in radial velocity over a range gate.

Figure 8 illustrates the returned power from the vertical beam in the sodar simulator, calculated as a 10-min average. This power is calculated as the peak value of a sum of all the instantaneous frequency spectra contained within the averaging period. We see the decrease in power with range, as expected due to the $1 / r^{2}$ term in (7). During the first 20 min of simulation, power values are elevated close to the boundary layer height due to increased spatial variation in temperature there. 

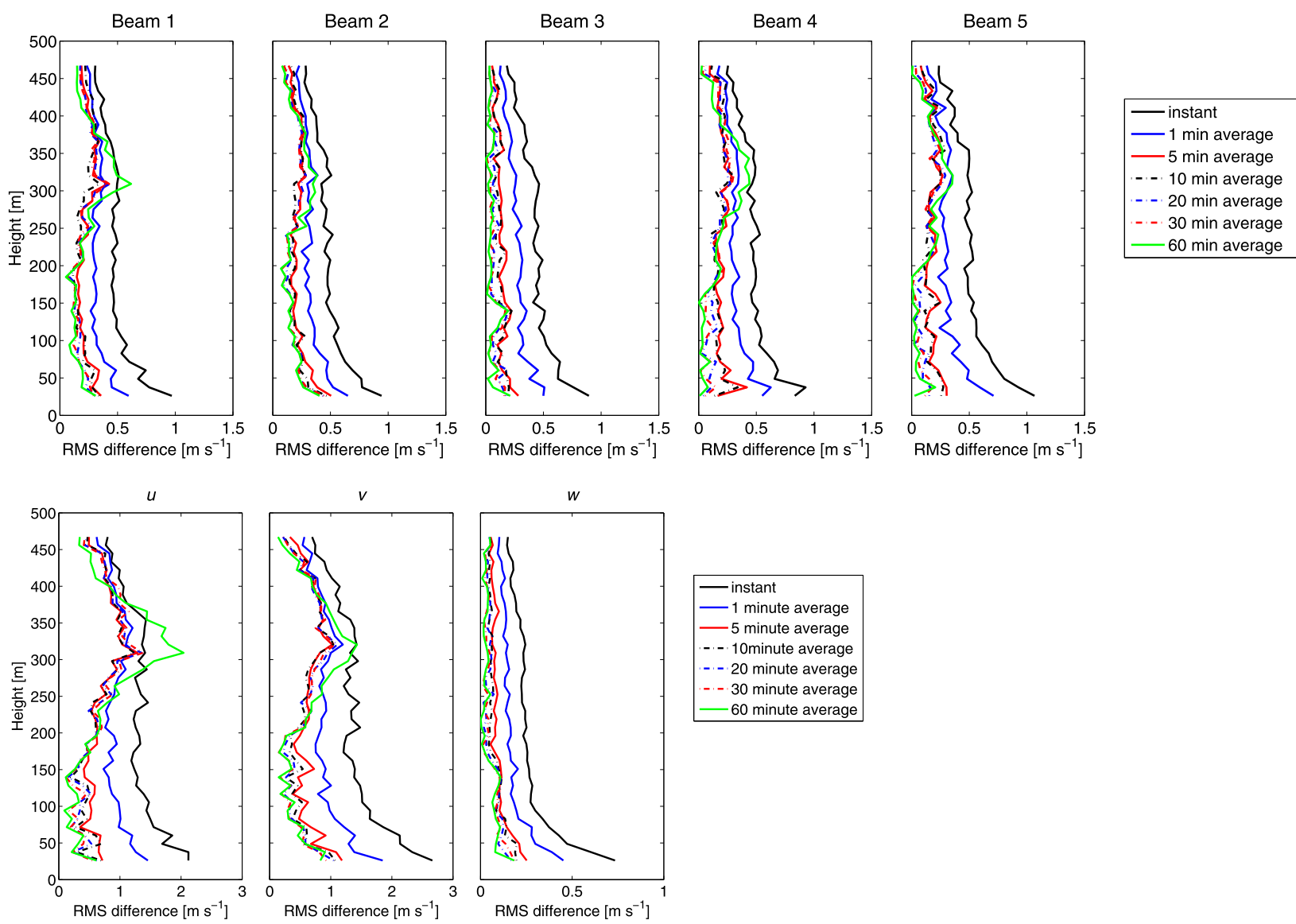

FIG. 7. (top) The RMS difference between radial velocity derived from the moment and spectral processing techniques, (middle) the vertical beam, (bottom) the resulting RMS differences in derived values of $u$, $v$, and $w$. The different colored lines represent different averaging periods.

The main goal of this study was to evaluate the recently developed time series sodar simulator using LES output. As discussed in section 3, the performance of operational sodars is often evaluated through comparison with in situ wind measurements. The simulator is evaluated in this manner in Fig. 9. The figure illustrates the wind speed and direction that would be calculated at five different heights $(40,100,200,300$, and $400 \mathrm{~m})$ corresponding to measurement levels on a virtual instrumented (i.e., fitted with sonic anemometers) tower whose base is exactly collocated with the simulated sodar. The simulated wind measurements from the sonic anemometers are taken from the LES data in the desired location, with no addition of instrument or background noise. The left panels show results from the moment simulator, and the differences between the moment simulator and virtual tower data can be interpreted as representative of the differences caused by point versus spatially averaged measurements. Clearly, the time series simulator (the right panels in Fig. 9) produces greater outliers than the moment simulator in both wind speed and direction. This is further evidenced by the lower $R^{2}$ values in both wind speed and direction from the time series simulator than the moment simulator (shown on Fig. 9). The top-right panel of Fig. 9 indicates that the time series sodar typically overestimates the wind speed as compared to the virtual tower, where the wind speed values are calculated directly from the LES. Errors in the spectrally estimated radial velocity caused by the assumption of stationarity of the sign may also be contributing to this effect. The wind speed generally increases with height, and as discussed above, a linear increase in radial velocity across a range gate can result in an increase in the spectrally derived radial velocity.

\section{Conclusions}

In this study, we designed a sodar simulator that ingests numerically simulated turbulent flow fields. The simulator has been developed in a way that many of its operating parameters are defined by the user in order to 


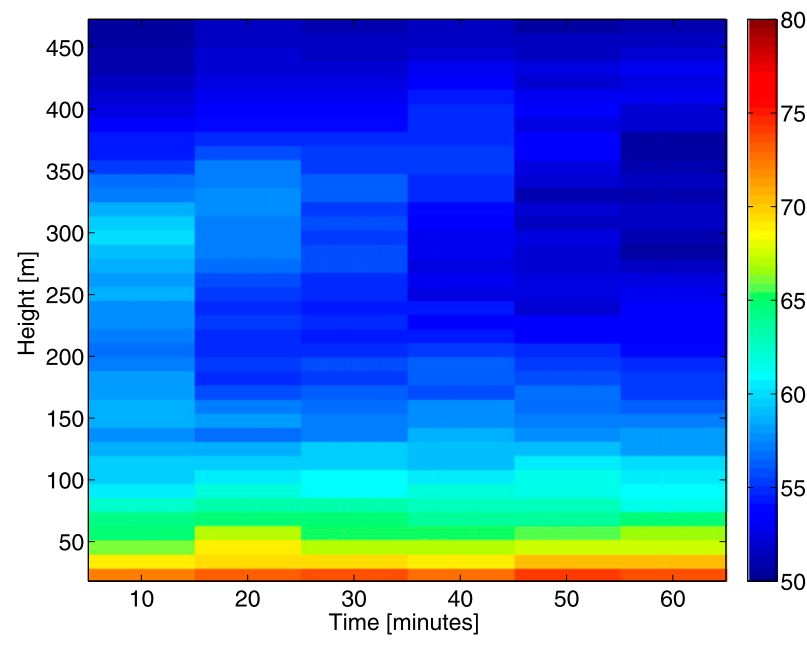

FIG. 8. Power from the vertical beam of the sodar simulator with an arbitrary set zero power value.

facilitate comparisons with operational systems. LES data from a clear CBL case were used in the study. To evaluate the time series simulator, a moment simulator was developed in parallel. The moment simulator uses the same beam locations as the time series simulator, but it does not include the spectral processing. In the moment simulator, the radial velocities contained within a range gate are simply averaged to provide a representative velocity value. The use of the moment simulator as a comparison tool ensures that any differences are due to the sodar measurement and signal processing technique and not caused by the invalidity of assuming horizontal homogeneity of the three-component wind field over the beams.

The performance of the time series simulator is consistent with the moment simulator. Once again, the RMS differences in $u$ and $v$ were highest at the boundary layer top, where the wind components change the most rapidly with height. As seen in Fig. 9, the derived wind speed values from the time series simulator tend to be higher than the ones measured at the virtual instrumented tower emulated through LES. We surmise that this is partially due to the effect of trilinear interpolation introducing an unphysical perfect linear variation in radial velocity across the range gates. Ways to mitigate this undesirable effect include the addition of subgrid turbulence kinetic energy, although poor results
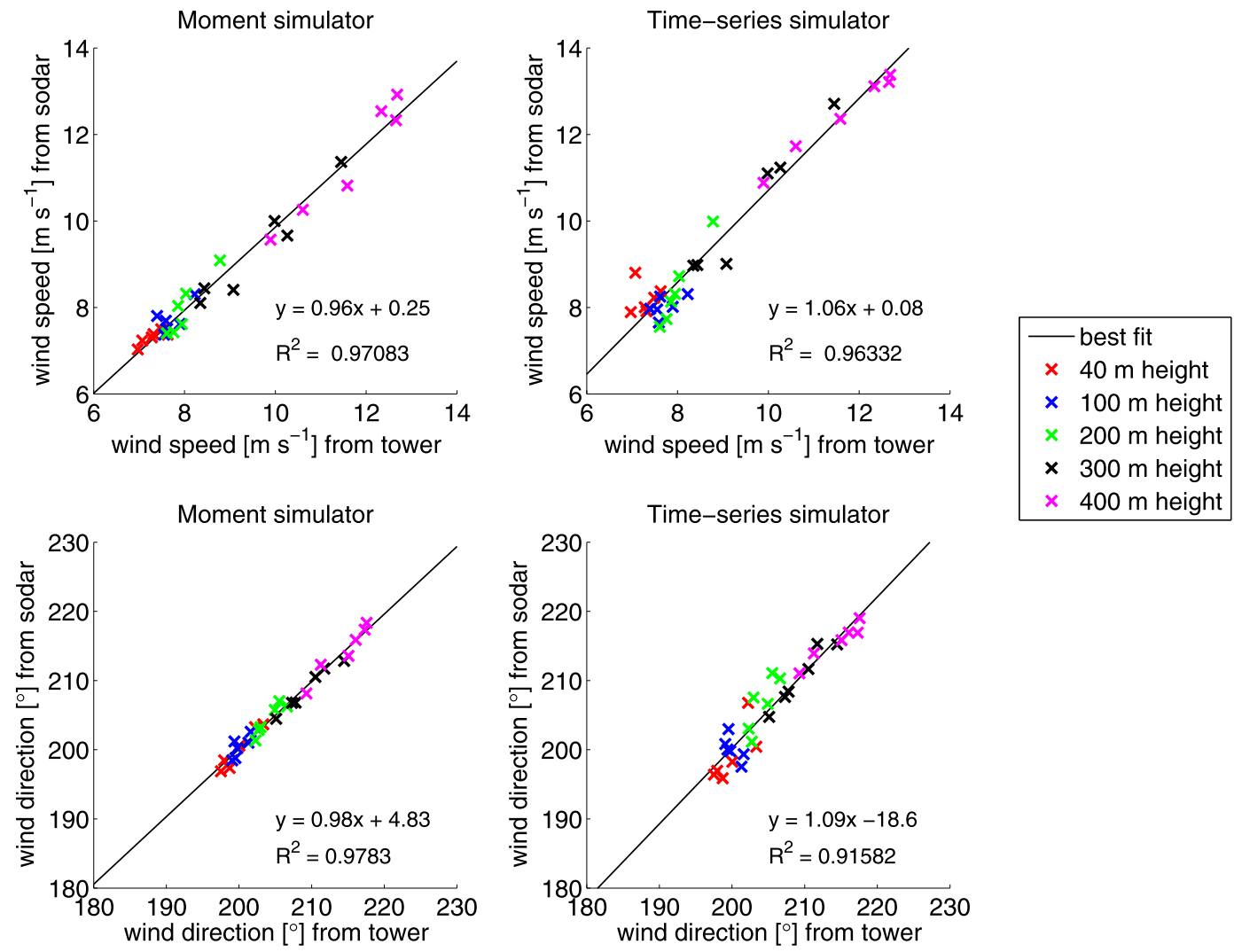

FIG. 9. A comparison of the derived 10-min averaged wind speeds and directions from a virtual instrumented tower, the moment simulator, and the time series simulator. The virtual tower data refer to five different heights, while the moment and time series simulators are spatially averaged. 
from the initial testing of this theory suggest that further investigation is required to determine an appropriate scaling factor to use when interpolating subgrid values down to scales considerably smaller than the grid spacing of the LES.

The returned power modeled by the developed sodar simulator appears realistic for a clear CBL case.

Overall, the sodar simulator provides a useful tool for investigation of the way the boundary layer is viewed by acoustic remote sensors. It allows for the emulation of operational sodar systems and for the testing of different scanning strategies and signal processing techniques on the same turbulent flow data. It also provides a way to compare wind data that would be obtained by point and spatially averaged measurements in a variety of simulated atmospheric environments.

Planned future work will be centered upon an investigation into an appropriate scaling for the inclusion of spatially interpolated values of resolved velocity and subgrid turbulence kinetic energy. This should allow for a reduction in the RMS differences in wind components between the moment- and spectrally derived values for $u, v$, and $w$. It is also planned to test the simulator on LES data for the stable boundary layer as well as to include additional features in the simulator, such as the modeling of sodar-observed turbulent fluctuations of the vertical velocity.

Acknowledgments. The National Science Foundation (NSF) is acknowledged for the support of the reported study through the Grant ATM-1016153. Three anonymous reviewers provided suggestions that substantially improved the manuscript.

\section{REFERENCES}

Antoniou, I., H. E. Joergensen, F. Ormel, S. Bradley, S. von Hünerbein, S. Emeis, and G. Warmbier, 2003: On the theory of sodar measurement techniques. Ris $\varnothing$ DTU National Laboratory Rep. Ris $\varnothing$-R-1410 (EN), 60 pp.

Balsley, B. B., and K. Gage, 1982: On the use of radars for operational profiling. Bull. Amer. Meteor. Soc., 63, 1009-1018, doi:10.1175/1520-0477(1982)063<1009:OTUORF>2.0.CO;2.

Benjamin, S. G., K. J. Brundage, and L. L. Morone, 1994: Implementation of the Rapid Update Cycle. Part I: Analysis/model description. NOAA/NWS Tech. Procedures Bull. 416, 16 pp.

Botnick, A. M., and E. Fedorovich, 2008: Large eddy simulation of atmospheric convective boundary layer with realistic environmental forcings. Quality and Reliability of Large-Eddy Simulations, J. Meyers, B. Geurts, and P. Sagaut, Eds., ERCOFTAC Series, Vol. 12, Springer Verlag, 193-204.

Bradley, S. G., 1999: Use of coded waveforms for sodar systems. Meteor. Atmos. Phys., 71, 15-23, doi:10.1007/s007030050039.

— 2008a: Atmospheric Acoustic Remote Sensing. CRC Press, 296 pp.
- 2008b: Wind speed errors for lidars and sodars in complex terrain. IOP Conf. Ser.: Earth Environ. Sci., 1, 012061, doi:10.1088/1755-1315/1/1/012061.

—, 2012: The noise part of sodar signal-to-noise. Extended Abstracts, 16th Int. Symp. for the Advancement of BoundaryLayer Remote Sensing, Boulder, CO, ISARS, 271-274. [Available online at http://www.esrl.noaa.gov/psd/events/2012/ isars/pdf/isars2012-abstractVolume.pdf.]

- 2013: Aspects of the correlation between sodar and mast instrument winds. J. Atmos. Oceanic Technol., 30, 2241-2247, doi:10.1175/JTECH-D-12-00256.1.

Capsoni, C., M. D'Amico, and R. Nebuloni, 2001: A multiparameter polarimetric radar simulator. J. Atmos. Oceanic Technol., 18, 1799-1809, doi:10.1175/1520-0426(2001)018<1799: AMPRS $>2.0 . \mathrm{CO} ; 2$.

Cheong, B. L., M. W. Hoffman, and R. D. Palmer, 2004: Efficient atmospheric simulation for high-resolution radar imaging applications. J. Atmos. Oceanic Technol., 21, 374-378, doi:10.1175/ 1520-0426(2004)021<0374:EASFHR > 2.0.CO;2.

- R. D. Palmer, and M. Xue, 2008: A time series weather radar simulator based on high-resolution atmospheric models. J. Atmos. Oceanic Technol., 25, 230-243, doi:10.1175/ 2007JTECHA923.1.

Clothiaux, E., T. Ackerman, and D. Babb, 1996: Ground-based remote sensing of cloud properties using millimeter-wave radar. Radiation and Water in the Climate System: Remote Measurements, E. Raschke, Ed., NATO ASI Series I, Vol. 45, Springer, 323-366.

Cook, D. R., and M. S. Pekour, 2008: Eddy correlation flux measurement system handbook. DOE Tech. Rep. DOE/SCARM/TR-05, 15 pp. [Available online at http://www.wmo int/pages/prog/gcos/documents/gruanmanuals/Z_instruments/ ecor_handbook.pdf.]

Crescenti, G. H., 1997: A look back on two decades of Doppler sodar comparison studies. Bull. Amer. Meteor. Soc., 78, 651673, doi:10.1175/1520-0477(1997)078<0651:ALBOTD>2.0.CO;2.

Deardorff, J. W., 1980: Stratocumulus-capped mixed layer derived from a three-dimensional model. Bound-Layer Meteor., 18, 495-527, doi:10.1007/BF00119502.

Fedorovich, E., F. T. M. Nieuwstadt, and R. Kaiser, 2001: Numerical and laboratory study of horizontally evolving convective boundary layer. Part I: Transition regimes and development of the mixed layer. J. Atmos. Sci., 58, 70-86, doi:10.1175/1520-0469(2001)058<0070:NALSOA $>2.0 . C O ; 2$.

— , R. Conzemius, and D. Mironov, 2004a: Convective entrainment into a shear-free, linearly stratified atmosphere: Bulk models reevaluated through large eddy simulations. J. Atmos. Sci., 61, 281-295, doi:10.1175/1520-0469(2004)061<0281: CEIASL $>2.0 . \mathrm{CO} ; 2$.

— , and Coauthors, 2004b: Entrainment into sheared convective boundary layers as predicted by different large eddy simulation codes. Preprints, 16th Symp. on Boundary Layers and Turbulence, Portland, ME, Amer. Meteor. Soc., P4.7. [Available online at https://ams.confex.com/ams/BLTAIRSE/ techprogram/paper_78656.htm.]

Gibbs, J. A., E. Fedorovich, and A. M. J. van Eijk, 2011: Evaluating Weather Research and Forecasting (WRF) model predictions of turbulent flow parameters in a dry convective boundary layer. J. Appl. Meteor. Climatol., 50, 2429-2444, doi:10.1175/ 2011JAMC2661.1.

Greenhut, G. K., and G. Mastrantonio, 1989: Turbulence kinetic energy budget profiles derived from Doppler sodar 
measurements. J. Appl. Meteor., 28, 99-106, doi:10.1175/ 1520-0450(1989)028<0099:TKEBPD>2.0.CO;2.

ISO, 1993: Acoustics-Attenuation of sound during propagation outdoors-Part 1: Calculation of the absorption of sound by the atmosphere. International Standards Organization Rep. ISO9613-1:1993(E), 26 pp.

Jung, Y., G. Zhang, and M. Xue, 2008: Assimilation of simulated polarimetric radar data for a convective storm using the ensemble Kalman filter. Part I: Observation operators for reflectivity and polarimetric variables. Mon. Wea. Rev., 136, 2228-2245, doi:10.1175/2007MWR2083.1.

Kallistratova, M. A., 1997: Physical grounds for acoustic remote sensing of the boundary layer. Acoustic Remote Sensing Application, S. P. Singal, Ed., Lecture Notes in Earth Sciences, Vol. 69, Springer Verlag, 3-34.

- and R. L. Coulter, 2004: Application of sodars in the study and monitoring of the environment. Meteor. Atmos. Phys., 85, 21-37.

Koscielny, A. J., R. J. Doviak, and D. S. Zrnić, 1984: An evaluation of the accuracy of some radar wind profiling techniques. J. Atmos. Oceanic Technol., 1, 309-320, doi:10.1175/ 1520-0426(1984)001<0309:AEOTAO > 2.0.CO;2.

Little, C. G., 1969: Acoustic methods for the remote probing of the lower atmosphere. Proc. IEEE, 57, 571-578, doi:10.1109/ PROC.1969.7010.

Masri, P., and A. Bateman, 1995: Identification of nonstationary audio signals using the FFT, with application to analysis-based synthesis of sound. IEEE Colloquium on Audio Engineering Digest, Vol. 1995/089, IEEE, 11/1-11/6.

May, P. T., R. G. Strauch, K. P. Moran, and W. L. Ecklund, 1990: Temperature sounding by RASS with wind profiler radars: A preliminary study. IEEE Trans. Geosci. Remote Sens., 28, 1928, doi:10.1109/36.45742.

May, R. M., M. I. Biggerstaff, and M. Xue, 2007: A Doppler radar emulator with an application to the detectability of tornadic signatures. J. Atmos. Oceanic Technol., 24, 19731996, doi:10.1175/2007JTECHA882.1.

Muschinski, A., P. P. Sullivan, D. B. Wuertz, R. J. Hill, S. A. Cohn, D. H. Lenschow, and R. J. Doviak, 1999: First synthesis of wind-profiler signals on the basis of large-eddy simulation data. Radio Sci., 34, 1437-1459, doi:10.1029/1999RS900090.

Neff, W. D., 1988: Observations of complex terrain flows using acoustic sounders: Echo interpretation. Bound-Layer Meteor., 42, 207-228, doi:10.1007/BF00123813.

Palmer, R. D., M. F. Larsen, E. L. Sheppard, S. Fukao, M. Yamamoto, T. Tsuda, and S. Kato, 1993: Poststatistic steering wind estimation in the troposphere and lower stratosphere. Radio Sci., 28, 261-271, doi:10.1029/93RS00279.

Pan, N., 2003: Excess attenuation of an acoustic beam by turbulence. J. Acoust. Soc. Amer., 114, 3102-3111, doi:10.1121/1.1628679.

Scipión, D. E., A. M. Botnick, R. D. Palmer, P. B. Chilson, and E. Fedorovich, 2008: Inter-comparison of retrieved wind fields from large-eddy simulations and radar measurements in the convective boundary layer. IOP Conf. Ser.: Earth Environ. Sci., 1, 012003, doi:10.1088/1755-1315/1/1/012003.

- , R. D. Palmer, P. B. Chilson, E. Fedorovich, and A. M. Botnick, 2009: Retrieval of convective boundary layer wind fields statistics from radar profiler measurements in conjunction with large eddy simulations. Meteor. Z., 18, 175-187, doi:10.1127/ 0941-2948/2009/0371

Soler, M. R., J. Hinojosa, M. Bravo, D. Pino, and J. V.-G. de Arellano, 2003: Analyzing the basic features of different complex terrain flows by means of a Doppler sodar and a numerical model: Some implications for air pollution problems. Meteor. Atmos. Phys., 85, 141-154.

Tatarskii, V. I., 1971: The Effects of the Turbulent Atmosphere on Wave Propagation. Kefer Press, 472 pp.

Yu, T.-Y., and R. D. Palmer, 2001: Atmospheric radar imaging using spatial and frequency diversity. Radio Sci., 36, 14931504, doi:10.1029/2000RS002622. 